\title{
ARENS REGULARITY AND THE ALGEBRA OF DOUBLE MULTIPLIERS
}

\author{
B. J. TOMIUK
}

\begin{abstract}
We show how Arens regularity of a w.c.c. Banach algebra $A$ with a bounded approximate identity is connected with the algebra of double multipliers on $A$.
\end{abstract}

1. Introduction. Let $A$ be a semisimple w.c.c. Banach algebra with a bounded approximate identity. Let $E$ be an element of the second conjugate space $A^{* *}$ such that $E$ is a left identity for $\left(A^{* *}, \circ^{\prime}\right)$ and a right identity for $\left(A^{* *}, \circ\right)$, where $\circ$ ' and $\circ$ denote the two Arens products on $A^{* *}$. Let $M(A)$ be the algebra of double multipliers on $A$. We show that $A$ is Arens regular if and only if $S^{* *}(E)=T^{* *}(E)$ for all $(S, T) \in M(A)$.

2. Preliminaries. Let $A$ be a Banach algebra. Then $A^{*}$ and $A^{* *}$ will denote the first and second conjugate spaces of $A$ and $\pi$ the canonical map of $A$ into $A^{* *}$. For any subset $S$ in $A, l(S)$ and $r(S)$ will denote, respectively, the left and right annihilators of $S$ in $A$. By an ideal we shall always mean a two-sided ideal unless otherwise specified.

For any $a \in A$, let $L_{a}$ and $R_{a}$ be, respectively, the left and right multiplication operators determined by $a$. An element $a \in A$ is called left weakly completely continuous (1.w.c.c.) if $L_{a}$ is weakly completely continuous. $A$ is called 1.w.c.c. if every $a \in A$ is l.w.c.c. Similarly an element $a \in A$ is called right weakly completely continuous (r.w.c.c.) if $R_{a}$ is weakly completely continuous, and $A$ is called r.w.c.c. if every $a \in A$ is r.w.c.c. If $A$ is both l.w.c.c. and r.w.c.c., then $A$ is called w.c.c.

A map $T: A \rightarrow A$ is called a left (resp. right) multiplier if $T(a b)=T(a) b$ (resp. $T(a b)=a T(b))$ for all $a, b \in A$. Let $M_{l}(a)$ (resp. $M_{r}(a)$ ) denote the algebra of all bounded linear left (resp. right) multipliers on $A$. For $a \in A, L_{a} \in M_{l}(A)$ and $R_{a} \in M_{r}(A)$.

An ordered pair $(S, T)$ of mappings from $A$ into itself is called a double multiplier if $x S(y)=T(x) y$, for all $x, y \in A$ [6]. Let $M(A)$ denote the algebra of double multipliers $(S, T)$ on $A$ such that $S \in M_{l}(A)$ and $T \in M_{r}(A)$. If $A$ is faithful, i.e., $l(A)=r(A)=(0)$, then every double multiplier on $A$ belongs to $M(A)$ [8, Lemma 3.1, p. 269].

Let $A$ be a Banach algebra. The two Arens products on $A^{* *}$ are defined in stages as follows. Let $x, y \in A, f \in A$ and $F, G \in A^{* *}$.

Received by the editors October 24, 1979 and, in revised form, February 21, 1980.

AMS (MOS) subject classifications (1970). Primary 46H20, 46H10; Secondary 46L2O.

Key words and phrases. Weakly completely continuous (w.c.c.) Banach algebra, Arens products, Arens regularity, double multipliers. 
Define $f \circ x \in A^{*}$ by $(f \circ x)(y)=f(x y)$.

Define $F \circ f \in A^{*}$ by $(F \circ f)(x)=F(f \circ x)$.

Define $F \circ G \in A^{* *}$ by $(F \circ G)(f)=F(G \circ f)$.

Define $x \circ{ }^{\prime} f \in A^{*}$ by $\left(x \circ{ }^{\prime} f\right)(y)=f(y x)$.

Define $f \circ{ }^{\prime} F \in A^{*}$ by $\left(f \circ{ }^{\prime} F\right)(x)=F\left(x \circ{ }^{\prime} f\right)$.

Define $F \circ \circ^{\prime} G \in A^{* *}$ by $\left(F \circ{ }^{\prime} G\right)(f)=G\left(f \circ{ }^{\prime} F\right)$.

Then $A^{* *}$ is a Banach algebra under the products $F \circ G$ and $F \circ{ }^{\prime} G$, and $\pi$ is an algebra isomorphism of $A$ into $\left(A^{* *}, \circ\right)$ and $\left(A^{* *}, \circ{ }^{\prime}\right)$.

A Banach algebra $A$ is called Arens regular if the two Arens products agree on $A^{* *}$. If a Banach algebra $A$ contains a bounded right approximate identity then $\left(A^{* *}, \circ\right)$ has a right identity and if $A$ contains a bounded left approximate identity then $\left(A^{* *}, \circ^{\prime}\right)$ has a left identity. (See [1, Lemma 3.3, p. 855].) If $A$ has a bounded approximate identity then $A^{* *}$ has an element $E$ which is simultaneously a right identity for $\left(A^{* *}, \circ\right)$ and a left identity for $\left(A^{* *}, \circ^{\prime}\right)$ [5, Proposition 1.3, p. 93]. It is an immediate consequence of the definition of Arens products and [2, Theorem 2, p. 482] that a Banach algebra $A$ is left (right) w.c.c. if and only if $\pi(A)$ is a right (left) ideal of $A^{* *}$ under either Arens product.

All algebras under consideration are over the complex field.

3. Direct sum decompositions of $A^{* *}$.

Notation. Let $A$ be a Banach algebra. Set

$$
N_{A}=\left\{G \in A^{* *}: \pi(x) \circ G=0 \text { for all } x \in A\right\}
$$

and

$$
N_{A}^{\prime}=\left\{G \in A^{* *}: G \circ{ }^{\prime} \pi(x)=0 \text { for all } x \in A\right\} .
$$

Let $R_{1}^{* *}$ be the radical of $\left(A^{* *}, \circ\right)$ and $R_{2}^{* *}$ the radical of $\left(A^{* *}, \circ{ }^{\prime}\right)$.

We observe that $\pi(x) \circ G=\pi(x) \circ{ }^{\prime} G$ and $G \circ \pi(x)=G \circ{ }^{\prime} \pi(x)$ for all $x \in A$ and $G \in A^{* *}[4]$. Although the following lemma can be easily deduced from the results in [8] stated for right multipliers, for the sake of completeness we sketch its proof.

LEMMA 3.1 Let $A$ be a Banach algebra with a bounded left approximate identity. Let $E$ be a left identity in $\left(A^{* *}, \circ^{\prime}\right)$. Then the following statements hold.

(i) For $S \in M_{l}(A)$, let $F^{S}=S^{* *}(E)$. Then the mapping $S \rightarrow F^{S}$ is a continuous algebra isomorphism of $M_{l}(A)$ into $\left(A^{* *}\right.$, $\left.{ }^{\prime}\right)$, and for each $S \in M_{l}(A)$,

$$
\pi(S(x))=F^{S} \circ{ }^{\prime} \pi(x) \quad(x \in A) .
$$

(ii) For each $F \in A^{* *}$ such that $F \circ^{\prime} \pi(A) \in \pi(A)$, there exists $S \in M_{l}(A)$ and $G \in N_{A}^{\prime}$ such that $F=S^{* *}(E)+G$.

Proof. (i) If in the argument in [7, p. 810] we replace the product * by the product $\circ^{\prime}$ and $T$ by $S \in M_{l}(A)$ and make the obvious corresponding changes, we will obtain

$$
f \circ{ }^{\prime} F^{S}=S^{*}(f) \quad\left(f \in A^{*}, S \in M_{l}(A)\right) .
$$

It is easy to see that the mapping $S \rightarrow F^{S}$ is linear and one-to-one. It is also 
continuous since

$$
\left\|F^{S}\right\|=\left\|S^{* *}(E)\right\| \leqslant\left\|S^{* *}\right\|\|E\|=\|S\|\|E\| .
$$

Let $S_{1}, S_{2} \in M_{l}(A)$ and $f \in A^{*}$. Then using (1), we have

$$
\begin{aligned}
\left(F^{\left.S_{1} \circ{ }^{\prime} F^{S_{2}}\right)(f)}\right. & =F^{S_{2}}\left(f \circ{ }^{\prime} F^{S_{1}}\right)=F^{S_{2}}\left(S_{1}^{*}(f)\right) \\
& =S_{2}^{* *}(E)\left(S_{1}^{*}(f)\right)=S_{1}^{* *}\left(S_{2}^{* *}(E)\right)(f) \\
& =\left(S_{1} S_{2}\right)^{* *}(E)(f)=F^{S_{1} S_{2}}(f) .
\end{aligned}
$$

Therefore $S_{1} S_{2} \rightarrow F^{S_{1}} \circ{ }^{\prime} F^{S_{2}}$. Let $x \in A, f \in A^{*}$ and $S \in M_{l}(A)$. Then, using (1) again, we have

$$
\begin{aligned}
S^{* *}(\pi(x))(f) & =\pi(x)\left(S^{*}(f)\right)=S^{*}(f)(x) \\
& =\left(f \circ{ }^{\prime} F^{S}\right)(x)=\pi(x)\left(f \circ{ }^{\prime} F^{S}\right) \\
& =\left(F^{S} \circ{ }^{\prime} \pi(x)\right)(f) .
\end{aligned}
$$

Hence

$$
\pi(S(x))=S^{* *}(\pi(x))=F^{S} \circ{ }^{\prime} \pi(x) \quad(x \in A) .
$$

(ii) Let $F \in A^{* *}$ such that $F \circ{ }^{\prime} \pi(A) \subset \pi(A)$. Then since $S \rightarrow S^{* *} \mid \pi(A)$ (the restriction of $S^{* *}$ to $\left.\pi(A)\right)$ is an isometric algebra isomorphism of $M_{l}(A)$ onto $M_{l}(\pi(A))$, there exists $S \in M_{l}(A)$ such that

$$
S^{* *}(\pi(x))=F \circ{ }^{\prime} \pi(x) \quad(x \in A) .
$$

Let $F^{S}=S^{* *}(E)$. By (i),

$$
F^{S} \circ{ }^{\prime} \pi(x)=S^{* *}(\pi(x))=\pi(S(x))=F \circ{ }^{\prime} \pi(x) \quad(x \in A) .
$$

Therefore $\left(F-F^{S}\right) \circ^{\prime} \pi(x)=0(x \in A)$ so that $G=F-F^{S} \in N_{A}^{\prime}$ and $F=F^{S}$ $+G$.

THEOREM 3.2. Let $A$ be a semisimple r.w.c.c. Banach algebra with a bounded left approximate identity. Let $E$ be a left identity of $\left(A^{* *}, \circ{ }^{\prime}\right)$. Then

(i) $A^{* *}=N_{A}^{\prime} \oplus M^{\prime}$, where $M^{\prime}=\left\{S^{* *}(E): S \in M_{l}(A)\right\}$.

(ii) $N_{A}^{\prime}=\left\{G \in A^{* *}: G \circ{ }^{\prime} E=0\right\}$ and $N_{A}^{\prime}=R_{2}^{* *}$.

(iii) $M^{\prime}=\left\{G \circ{ }^{\prime} E: G \in A^{* *}\right\}$ and $M^{\prime}$ is a closed left ideal of $\left(A^{* *}\right.$, $\left.\circ\right)$.

Proof. (i) Since $A$ is r.w.c.c., $\pi(A)$ is a left ideal of $\left(A^{* *}\right.$, ० $)$ so that $F \circ{ }^{\prime} \pi(A)$ $\subset \pi(A)$ for every $F \in A^{* *}$. Therefore $A^{* *}=N_{A}^{\prime}+M^{\prime}$ by Lemma 3.1. Let $F \in$ $N_{A}^{\prime} \cap M^{\prime}$. Then $F=S^{* *}(E)$ for some $S \in M_{l}(A)$ and $\pi(S(x))=F \circ{ }^{\prime} \pi(x)=0$ for all $x \in A$. Therefore $S=0$ and hence $F=0$. Thus $A^{* *}=N_{A}^{\prime} \oplus M^{\prime}$.

(ii) Since $G \circ^{\prime} F$ is $w^{*}$-continuous in $F$ for fixed $G[4$, p. 116] and $\pi(A)$ is $w^{*}$-dense in $A^{* *}, N_{A}^{\prime}=\left\{G \in A^{* *}: G \circ{ }^{\prime} A^{* *}=0\right\}$ so that $N_{A}^{\prime}$ is an ideal of $\left(A^{* *}\right.$, ○ '). Clearly $N_{A}^{\prime} \subset R_{2}^{* *}$. By [3, Theorem 4.6, p. 130], $R_{2}^{* *} \cap \pi(A)=(0)$ and, by hypothesis, $\pi(A)$ is a left ideal of $\left(A^{* *}\right.$, ○'). Hence $R_{2}^{* *} \circ^{\prime} \pi(A) \subset R_{2}^{* *} \cap \pi(A)$ $=(0)$. Therefore $R_{2}^{* *} \subset N_{A}^{\prime}$. Thus $N_{A}^{\prime}=R_{2}^{* *}$. That $N_{A}^{\prime} \subset\left\{G \in A^{* *}: G \circ{ }^{\prime} E=0\right\}$ is clear. On the other hand if $G \circ^{\prime} E=0$ then $G \circ^{\prime} \pi(x)=G \circ^{\prime}\left(E \circ \circ^{\prime} \pi(x)\right)=$ $\left(G \circ{ }^{\prime} E\right) \circ^{\prime} \pi(x)=0$ for all $x \in A$ and hence $G \in N_{A}^{\prime}$.

(iii) Let $F=S^{* *}(E) \in M^{\prime}$. Then $F \circ^{\prime} E=S^{* *}(E) \circ^{\prime} E$ and, since $S^{* *}$ is a left 
multiplier on $A^{* *}$, we get $S^{* *}(E) \circ^{\prime} E=S^{* *}\left(E \circ^{\prime} E\right)=S^{* *}(E)=F$. Conversely suppose that $F=G \circ^{\prime} E, G \in A^{* *}$. Then there are $S \in M_{l}(A)$ and $Q \in N_{A}^{\prime}$ such that $F=S^{* *}(E)+Q$. Hence $F \circ^{\prime} E=S^{* *}(E) \circ^{\prime} E+Q \circ^{\prime} E=S^{* *}(E) \circ{ }^{\prime} E$ $=S^{* *}(E)$. As $F \circ^{\prime} E=F$, we get $F=S^{* *}(E)$. Hence $M^{\prime}=\left\{G \circ{ }^{\prime} E: G \in\right.$ $\left.A^{* *}\right\}$. To see that $M^{\prime}$ is a left ideal of $\left(A^{* *}\right.$, ० '), let $F \in A^{* *}$ and let $S_{1} \in M_{l}(A)$ be such that $F=S_{1}^{* *}(E)+G, G \in N_{A}^{\prime}$. Then, for every $S \in M_{l}(A)$, we have $F \circ{ }^{\prime} S^{* *}(E)=S_{1}^{* *}(E) \circ{ }^{\prime} S^{* *}(E)$. Since $S_{1}^{* *}$ is a left multiplier on $\left(A^{* *}, \circ^{\prime}\right)$ we obtain

$$
\begin{aligned}
S_{1}^{* *}(E) \circ^{\prime} S^{* *}(E) & =S_{1}^{* *}\left(E \circ{ }^{\prime} S^{* *}(E)\right)=S_{1}^{* *}\left(S^{* *}(E)\right) \\
& =S_{1}^{* *} S^{* *}(E)=\left(S_{1} S\right)^{* *}(E) \in M^{\prime}
\end{aligned}
$$

That $M^{\prime}$ is closed follows from the fact that $S \rightarrow S^{* *}(E)$ is a homeomorphism of $M_{l}(A)$ onto $M^{\prime}$ by Lemma 3.1 and a well-known theorem of Banach.

For an l.w.c.c. Banach algebra, Theorem 3.2 takes the following form (see also [9, p. 11]).

THeOREM 3.2'. Let A be a semisimple l.w.c.c. Banach algebra with a bounded right approximate identity. Let $E$ be a right identity in $\left(A^{* *}, \circ\right)$. Then

(i) $A^{* *}=N_{A} \oplus M$, where $M=\left\{T^{* *}(E): T \in M_{r}(A)\right\}$.

(ii) $N_{A}=\left\{G \in A^{* *}: E \circ G=0\right\}$ and $N_{A}=R_{1}^{* *}$.

(iii) $M=\left\{E \circ G: G \in A^{* *}\right\}$ and $M$ is a closed right ideal of $\left(A^{* *}, \circ\right)$.

COROLlary 3.3. Let $A$ be a semisimple w.c.c. Banach algebra with a bounded approximate identity. Then $R_{1}^{* *}=R_{2}^{* *}$.

Proof. Let $E$ be an element of $A^{* *}$ which is both a left identity for $\left(A^{* *}, \circ^{\prime}\right)$ and a right identity for $\left(A^{* *},{ }^{\circ}\right)$. Let $\left\{e_{\alpha}: \alpha \in \Omega\right\}$ be a bounded approximate identity in $A$. We may assume that $w^{*}-\lim _{\alpha} \pi\left(e_{\alpha}\right)=E$. Let $F \in R_{2}^{* *}$. Since $R_{2}^{* *}$ is an ideal of $\left(A^{* *}\right.$, $\left.{ }^{\prime}\right)$, we have that for all $x \in A, \pi(x) \circ{ }^{\prime} F \in R_{2}^{* *}$ and therefore, by Theorem 3.2 ,

$$
\left(\pi(x) \circ \circ^{\prime} F\right) \circ \circ^{\prime} E=0 \quad(x \in A) .
$$

But, since $A$ is w.c.c., $\pi(x) \circ^{\prime} F \in \pi(A)$ so that

$$
\left(\pi(x) \circ{ }^{\prime} F\right) \circ{ }^{\prime} E=(\pi(x) \circ F) \circ E=\pi(x) \circ F \quad(x \in A) .
$$

Hence $\pi(x) \circ F=0$ for all $x \in A$; in particular $\pi\left(e_{\alpha}\right) \circ F=0$ for all $\alpha \in \Omega$. Therefore, by the left $w^{*}$-continuity of the product $\circ$, we obtain that $E \circ F=0$. Hence $F \in R_{1}^{* *}$. Similarly we can show that if $F \in R_{1}^{* *}$ then $F \in R_{2}^{* *}$. Therefore $R_{1}^{* *}=R_{2}^{* *}$.

\section{The main result.}

LEMMA 4.1. Let $A$ be a semisimple Banach algebra with a bounded approximate identity. Let $E$ be an element of $A^{* *}$ which is both a left identity for $\left(A^{* *}, \circ\right)$ and a right identity for $\left(A^{* *}, \circ\right)$. Let $(S, T) \in M(A)$. Then

$$
S^{* *}(E)=T^{* *}(E)+G \text { where } G \in N_{A} .
$$


Proof. Let $F=S^{* *}(E)$. Then, by Lemma 3.1, we have $S^{* *}(\pi(x))=F \circ \pi(x)$ for all $x \in A$. Since

$$
T^{* *}(\pi(x)) \circ \pi(y)=\pi(x) \circ S^{* *}(\pi(y))=\pi(x) \circ F \circ \pi(y),
$$

for all $x, y \in A$, the semisimplicity of $A$ implies that

$$
T^{* *}(\pi(x))=\pi(x) \circ F \quad(x \in A) .
$$

Therefore, by [8, Lemma 5.3, p. 271], $F=T^{* *}(E)+G$, where $G \in N_{A}$.

ReMARK. Lemma 4.1 corrects an error in [8, Theorem 5.6, p. 272].

Theorem 4.2. Let $A$ be a semisimple w.c.c. Banach algebra with a bounded approximate identity. Let $E$ be an element of $A^{* *}$ which is both a left identity for $\left(A^{* *}, \circ\right)$ and a right identity for $\left(A^{* *}, \circ\right)$. Then $A$ is Arens regular if and only if $S^{* *}(E)=T^{* *}(E)$ for all $(S, T) \in M(A)$.

Proof. Suppose that $A$ is Arens regular. Then $A^{* *}$ is semisimple by [9, Corollary 6.2, p. 12]. Therefore, $R_{1}^{* *}=R_{2}^{* *}=(0)$ so that, by Lemma 4.1 and Theorem $3.2^{\prime}$, $S^{* *}(E)=T^{* *}(E)$ for every $(S, T) \in M(A)$.

Conversely suppose that $S^{* *}(E)=T^{* *}(E)$ for all $(S, T) \in M(A)$. By Theorems 3.2 and $3.2^{\prime}$ (and their notation), we have

$$
A^{* *}=R_{1}^{* *} \oplus M=R_{2}^{* *} \oplus M^{\prime} .
$$

By Corollary 3.3, $R_{1}^{* *}=R_{2}^{* *}$. We now show that $M=M^{\prime}$. Let $T \in M_{r}(A)$. Then, by $[8$, Lemma 5.1, p. 270],

$$
\pi(T(x))=T^{* *}(\pi(x))=\pi(x) \circ T^{* *}(E) \quad(x \in A) .
$$

Since $\pi(A)$ is a left ideal of $\left(A^{* *},{ }^{\circ}\right)$, there exists $S \in M_{l}(A)$ such that

$$
\pi(S(x))=T^{* *}(E) \circ \pi(x) \quad(x \in A) .
$$

Since $\pi(x S(y))=\pi(T(x) y)$ for all $x, y \in A$, we have that $(S, T) \in M(A)$. By hypothesis, $S^{* *}(E)=T^{* *}(E)$. Therefore $M \subset M^{\prime}$. Similarly we can show that for every $S \in M_{l}(A)$ there exists $T \in M_{r}(A)$ such that $S^{* *}(E)=T^{* *}(E)$ and $(S, T)$ $\in M(A)$. Therefore $M^{\prime} \subset M$. Hence $M=M^{\prime}$.

Now let $F_{1}, F_{2} \in M$. Let $\left(S_{1}, T_{1}\right)$ and $\left(S_{2}, T_{2}\right)$ be the elements of $M(A)$ defined by $F_{1}$ and $F_{2}$, respectively. That is, $\pi\left(S_{i}(x)\right)=F_{i} \circ \pi(x)$ and $\pi\left(T_{i}(x)\right)=\pi(x) \circ F_{i}$ for all $x \in A$ and $i=1,2$. We have that $S_{1} S_{2}$ is defined by $F_{1} \circ{ }^{\prime} F_{2}$ and $T_{2} T_{1}$ is defined by $F_{1} \circ F_{2}$. Since $\left(S_{1} S_{2}, T_{2} T_{1}\right) \in M(A)[8$, p. 270], it follows that

$$
\pi(x) \circ\left(F_{1} \circ{ }^{\prime} F_{2}\right) \circ \pi(y)=\pi(x) \circ\left(F_{1} \circ F_{2}\right) \circ \pi(y) \quad(x, y \in A) .
$$

Let $\left\{e_{\alpha}: \alpha \in \Omega\right\}$ be a bounded approximate identity in $A$. Since $\pi(x) \circ\left(F_{1} \circ{ }^{\prime} F_{2}\right)$ and $\pi(x) \circ\left(F_{1} \circ F_{2}\right)$ are in $\pi(A)$, letting $y=e_{\alpha}$ and taking the limit over $e_{\alpha}$, we obtain

$$
\pi(x) \circ\left(F_{1} \circ F_{2}\right)=\pi(x) \circ\left(F_{1} \circ F_{2}\right) \quad(x \in A) .
$$

Therefore $F_{1} \circ{ }^{\prime} F_{2}-F_{1} \circ F_{2} \in R_{1}^{* *}$. As $F_{1} \circ{ }^{\prime} F_{2}-F_{1} \circ F_{2} \in M$ and $M \cap R_{1}^{* *}$ $=(0)$, we see that

$$
F_{1} \circ{ }^{\prime} F_{2}=F_{1} \circ F_{2}
$$

so that the two Arens products agree on $M$. As they clearly agree on $R_{1}^{* *}$ and as $A^{* *}=R_{1}^{* *} \oplus M$, it follows that they agree on $A^{* *}$. Therefore $A$ is Arens regular. 
COROllary 4.3. Let $A$ be a semisimple w.c.c. Banach algebra with a bounded approximate identity. Then the following statements are equivalent.

(i) $A$ is Arens regular.

(ii) $\left(A^{* *}, \circ\right)$ is semisimple.

(iii) $\left(A^{* *}, \circ '\right)$ is semisimple.

Proof. If $A$ is Arens regular then, by [9, Corollary 6.2, p. 12], $A^{* *}$ is semisimple in each of the Arens products. By Corollary $3.3, R_{1}^{* *}=R_{2}^{* *}$ so that $\left(A^{* *}, \circ\right)$ is semisimple if and only if $\left(A^{* *}, \circ^{\prime}\right)$ is semisimple. If $R_{1}^{* *}=R_{2}^{* *}=(0)$ then $S^{* *}(E)$ $=T^{* *}(E)$ for all $(S, T) \in M(A)$. Therefore, by Theorem 4.2, $A$ is Arens regular.

It follows from the results above that if $G$ is an infinite compact abelian group then the group algebra $L^{1}(G)$ is not Arens regular. In fact $A=L^{1}(G)$ is a w.c.c. semisimple Banach algebra with a bounded approximate identity [10]. From [3, Theorem 5.5, p. 136] and Corollary 3.3 we obtain that $R_{1}^{* *}=R_{2}^{* *} \neq(0)$. Hence, by Corollary 4.3, $A$ is not Arens regular.

\section{REFERENCES}

1. P. Civin and B. Yood, The second conjugate space of a Banach algebra as an algebra, Pacific J. Math. 11 (1961), 847-870.

2. N. Dunford and J. T. Schwartz, Linear operators. Part I: General theory, Pure and Appl. Math., vol. 7, Interscience, New York and London, 1958.

3. S. L. Gulick, Commutativity and ideals in the biduals of topological algebras, Pacific J. Math. 18 (1966), 121-137.

4. J. Hennefeld, A note on the Arens products, Pacific J. Math. 26 (1968), 115-119.

5. Finding a maximal subalgebra on which the two Arens products agree, Pacific J. Math. 59 (1975), 93-98.

6. B. E. Johnson, An introduction to the theory of centralizers, Proc. London Math. Soc. (3) 14 (1964), 299-320.

7. L. Maté, Embedding multiplier operators of a Banach algebra $B$ into its second conjugate space $B^{* *}$, Bull. Acad. Polon. Sci. Sér. Sci. Math. Astronom. Phys. 13 (1965), 809-812.

8. B. J. Tomiuk, Multipliers on Banach algebras, Studia Math. 54 (1976), 267-283.

9. , On some properties of Segal algebras and their multipliers, Manuscripta Math. 27(1979), $1-18$.

10. B. J. Tomiuk and B. Yood, Topological algebras with dense socle, J. Functional Analysis 28 (1978), 254-277.

Department of Mathematics, University of Ottawa, Ottawa, Kin 6N5 Ontario, Canada 